Internetportal der Deutschen Krebsgesellschaft e.V.

dkg-web gmbh

Janett Lange

Straße des 17. Juni 106-108, 10623 Berlin, Germany

Tel.: +49 $30810316-112$

janett.lange@dkg-web.de

\title{
Monatsthema November: Sterbebegleitung - den letzten Weg gemeinsam gehen
}

Vielen Menschen in unserem Kulturkreis macht der Gedanke an Tod und Sterben große Angst. Umso wichtiger ist in dieser Situation das Zusammenspiel von unterstützender Begleitung, professioneller Pflege und medizinischer Betreuung. Dies entlastet Krebspatienten und ihre Angehörige und schafft Raum für eine gemeinsame Gestaltung der verbleibenden Zeit.

Umfragen zufolge möchte ein Großteil der Menschen die letzten Wochen und Tage am liebsten in vertrauter Umgebung zu Hause verbringen. Dies ist prinzipiell auch bei einer Krebserkrankung möglich. Wichtig dabei ist allerdings, dass eine oder mehrere Personen maßgeblich Verantwortung übernehmen und zudem die räumlichen Voraussetzungen für die Begleitung eines schwerkranken Patienten geeignet sind. Mit der häuslichen Betreuung eines Krebspatienten werden Angehörige nicht allein gelassen. Neben der $\mathrm{Zu}$ - sammenarbeit mit dem Hausarzt ist es ratsam, rechtzeitig auch Kontakt zu Klinikärzten und Sozialdiensten aufzunehmen. Bei der konkreten Umsetzung helfen außerdem verschiedene mobile Dienste. Wenn die Voraussetzungen für eine häusliche Pflege nicht mehr gegeben sind, bieten stationäre Hospize die Möglichkeit einer Betreuung in wohnlicher Atmosphäre.

Das aktuelle Experteninterview auf dem DKG-Internetportal beantwortet die wichtigsten Fragen zur ambulanten Palliativversorgung aus Sicht der Betroffenen und ihrer Angehörigen: Was können Angehörige tun? Worauf sollten Familienmitglieder vorbereitet sein und wer trägt eigentlich die Kosten für einen ambulanten Hospizdienst? Das gesamte Interview und viele hilfreiche Tipps und Infos zum Thema stehen $\mathrm{ab}$ sofort bereit unter www.krebsgesellschaft.de/ thema_november2012.

\section{KARGER}

Fax +497614520714 (c) 2012 S. Karger GmbH, Freiburg 